\title{
Human Umbilical Cord Blood-Derived Mesenchymal Stem Cell Therapy Promotes Functional Recovery of Contused Rat Spinal Cord through Enhancement of Endogenous Cell Proliferation and Oligogenesis
}

\author{
Sang In Park, ${ }^{1,2}$ Jung Yeon Lim, ${ }^{2}$ Chang Hyun Jeong, ${ }^{2}$ Seong Muk Kim, ${ }^{2}$ \\ Jin Ae Jun, ${ }^{2}$ Sin-Soo Jeun, ${ }^{2}$ and Won $\mathrm{Il} \mathrm{Oh}^{3}$ \\ ${ }^{1}$ Institute of Catholic Integrative Medicine (ICIM), Incheon St. Mary's Hospital, The Catholic University of Korea, \\ Incheon, Republic of Korea \\ ${ }^{2}$ Department of Neurosurgery, The Catholic University of Korea, Seoul, Republic of Korea \\ ${ }^{3}$ Medipost Biomedical Research Institute, Medipost Co., Ltd., Seoul, Republic of Korea
}

Correspondence should be addressed to Sin-Soo Jeun, ssjeun@catholic.ac.kr

Received 23 June 2011; Accepted 29 September 2011

Academic Editor: Ken-ichi Isobe

Copyright ( 12012 Sang In Park et al. This is an open access article distributed under the Creative Commons Attribution License, which permits unrestricted use, distribution, and reproduction in any medium, provided the original work is properly cited.

\begin{abstract}
Numerous studies have shown the benefits of mesenchymal stem cells (MSCs) on the repair of spinal cord injury (SCI) model and on behavioral improvement, but the underlying mechanisms remain unclear. In this study, to investigate possible mechanisms by which MSCs contribute to the alleviation of neurologic deficits, we examined the potential effect of human umbilical cord blood-derived MSCs (hUCB-MSCs) on the endogenous cell proliferation and oligogenesis after SCI. SCI was injured by contusion using a weight-drop impactor and hUCB-MSCs were transplanted into the boundary zone of the injured site. Animals received a daily injection of bromodeoxyuridine (BrdU) for 7 days after treatment to identity newly synthesized cells of ependymal and periependymal cells that immunohistochemically resembled stem/progenitor cells was evident. Behavior analysis revealed that locomotor functions of hUCB-MSCs group were restored significantly and the cavity volume was smaller in the MSCstransplanted rats compared to the control group. In MSCs-transplanted group, TUNEL-positive cells were decreased and BrdUpositive cells were significantly increased rats compared with control group. In addition, more of BrdU-positive cells expressed neural stem/progenitor cell nestin and oligo-lineage cell such as NG2, CNPase, MBP and glial fibrillary acidic protein typical of astrocytes in the MSC-transplanted rats. Thus, endogenous cell proliferation and oligogenesis contribute to MSC-promoted functional recovery following SCI.
\end{abstract}

\section{Introduction}

Recovery following spinal cord injury (SCI) is limited because of axonal damage [1], demyelination, and scar formation [2]. In addition to the formation of a central hemorrhagic lesion devoid of normal neurons and glia, oligodendrocytes and astrocytes in the white matter near the impact site are reduced by about $50 \%$ by $24 \mathrm{~h}$ after injury [3].

Recently, the use of stem cell for neurodegenerative disease has been widely investigated as a therapeutic strategy [4-6]. Neural stem cells have been used for the treatment of neurological diseases such as SCI [7] or stroke [8].
Numerous studies have reported that the survival and differentiation of grafted cells into neural cells correlate with behavior improvement. However, these cells are limited for clinical application because of insufficient cell supply, risk of immune rejection, and ethical problems. Since mesenchymal stem cells (MSCs) can be readily isolated and their numbers increased in vitro and differentiated into several types of mature cells including neurons, adipocytes, cartilage, and skeletal hepatocytes under appropriate conditions [9], a new therapeutic strategy has been a valuable source for central nervous stem (CNS) disease [10, 11]. Human umbilical cord blood-derived MSCs (hUCB-MSCs) have therapeutic 
potential and are attractive because these cells are readily available and are less immunogenic as compared to other sources of stem cells, such as bone marrow or adipose [12].

An alternative strategy of stem cell therapy is protection of injured cells and promotion of endogenous cell regeneration. Several studies have reported that stem cells might provide a better environment for damaged tissue and save remaining neurons by neurotrophic factors or cytokines [13, 14]. However, the specific mechanism of the MSCs for these assertions remains controversial and ill-explored. Nevertheless, MSC treatment of SCI has been reported as a candidate that supplies angiogenic, antiapoptotic, and mitogenic factors as well as migration toward damaged tissue [15]. Recently, MSCs have been used in clinical treatment and were shown to be effective in the treatment of various pathologies although evidence for distinct therapeutic mechanism was lacking [16].

The normal spinal cord contains endogenous neural progenitor cells (NPC) and oligodendrocyte precursor cells (OPCs) [17]. Nevertheless, production of new neurons and oligodendrocytes by endogenous cells into the spinal cord may be very restricted after injury [18]. Furthermore, cell transplantation studies have demonstrated that exogenous stem cells differentiate only very poorly when grafted into the spinal cord. Thus, the environment of the spinal cord appears to be highly restrictive for the differentiation of OPCs. If this environmental restriction can be changed by hUCBMSC in SCI, OPCs may be able to supply new neurons and oligodendrocytes. However, it is not known whether survival and differentiation generated from endogenous cells are influenced by transplanted hUCB-MSCs.

In the present study, we show that the transplantation of hUCB-MSCs confers therapeutic effects in a rat experimental SCI model. We investigated whether transplantation of hUCB-MSCs improved the functional recovery and improved the proliferation and genesis of resident endogenous cells within the spinal cord by hUCB-MSCs.

\section{Materials and Methods}

2.1. Human UCB-Derived MSCs. Human UCBs were obtained from normal full-term pregnant woman. The protocol for human subjects adhered to the guidelines outlined by the institutional review IRB board of the Catholic University of Korea (Seoul, Republic of Korea). hUCB-MSCs were isolated and expanded using a previously described protocol [12].

2.2. Animal Model. All animal protocols were approved by the Institutional Animal Care and Use Committee of Catholic University Medical School. Forty-five adult male SpragueDawley rats weighting between 270 and 300 g were employed in our experiments. Surgical techniques were similar to those described previously [19]. Briefly, rats were deeply anesthetized with ketamine-xylazine cocktail $(80 \mathrm{mg} / \mathrm{kg}$ of ketamine, $10 \mathrm{mg} / \mathrm{kg}$ of xylazine). Under a dissecting microscope, the skin and muscles overlying the thoracic cord were separated and retracted, the T9 vertebral level was removed by laminectomy, and the underlying spinal cord segment was exposed by slitting the dural sheath. The impact rod of the NYU impactor was centered above T9 and dropped from a height of $25 \mathrm{~mm}$ to induce an incomplete partial SCI. Following lesion, the dorsal back musculature was sutured and the skin closed with surgical clips. After surgery, the animals were kept on a thermostatically regulated heating pad until completely awake. The urinary bladder of all rats was emptied manually two times per day until recovery of urinary function.

2.3. Cell Transplantation. Rats were assigned randomly to one of the following two major groups: one group of rats were treated with $5 \mu \mathrm{L}$ phosphate-buffered saline (PBS) as the control group. The second group of rats received transplanted with hUCB-MSCs $\left(3 \times 10^{5}\right.$ cells $\left./ 5 \mu \mathrm{L}\right)$. In experiment 1 , the hUCB-MSCs was designed to test the therapeutic effectiveness $(n=26)$, and, in experiment 2 , these cells were designed to evaluate the proliferation of endogenous cells after transplantation $(n=12)$.

Initial locomotor scores were equalized between groups. The weight-drop injury level was based on our experience with the model to produce spontaneous recovery at a BassoBeatti-Bresnahan (BBB) score of 4 . Once the 46 rats were subjected to contusion SCI, they were divided randomly into the two groups. Using a 25-gauge syringe (Hamilton, Reno, NV) fixed in a stoelting stereotaxic frame (Dae Jong) at 7 days after injury, hUCB-MSCs were transplanted into the spinal cord $(0.5 \mathrm{~mm}$ from the midline, $1.5 \mathrm{~mm}$ down from the dura, and $5 \mathrm{~mm}$ rostral from the contusion epicenter). Each rat received a $5 \mu \mathrm{L}$ injection in the contusion site over a $10 \mathrm{~min}$ period each time. The cannula of the Hamilton syringe was left in place after injection for an additional $5 \mathrm{~min}$. All animal received antibiotics (Gentamicin sulfate, $30 \mathrm{mg} / \mathrm{kg} /$ day) during the first week after transplantation.

\subsection{5-Bromo-2-Deoxyuridine (BrdU) Administration. Spra-} gue-Dawley rats $(n=12)$ were injected with $50 \mathrm{mg} / \mathrm{kg}$ BrdU (Sigma-Aldrich, St. Louis, MO, USA) intraperitoneally each day for 7 days to label the newly generating cells after transplantation. The examined proliferative response focused on cell genesis occurring within 7 days after transplantation.

2.5. Behavioral Testing. The motor function restoration after spinal cord contusion was observed by open-field BBB locomotor ratio scale [20]. The scale used for measuring hind-limb function with these procedures ranges from a score of 0 , indicating no spontaneous movement, to a maximum score of 21 , with an increasing score indicating the use of individual joints, coordinated joint movement, coordinated limb movement, weight-bearing, and other functions. Behavioral testing was performed weekly on each hindlimb from the postoperative day to 7 weeks after SCI. Spinal cord contusion and cell transplantation were separately performed in double-blinded experiments by different investigators.

2.6. Tissue of Harvest. To study functional recovery and differentiation of transplanted hUCB-MSCs, rats from each group were sacrificed at 1 and 2 weeks (PBS, $n=3$; Transplantation, $n=3$ ) after transplantation and the others were 
examined by the BBB locomotor test 6 weeks after transplantation (PBS, $n=7$; transplantation, $n=7$ ). Also, to study endogenous cell proliferation after transplantation, rats from each group were sacrificed at $2 \mathrm{~h}$ and 1 week after the last BrdU injection $(n=5)$. All the rats were deeply anesthetized with a ketamine-xylazine cocktail $(80 \mathrm{mg} / \mathrm{kg}$ of ketamine, $10 \mathrm{mg} / \mathrm{kg}$ of xylazine) and then perfused transcardially with $0.01 \mathrm{M}$ PBS ( $\mathrm{pH}$ 7.4), followed by $4 \%$ paraformaldehyde (PFA) in $0.01 \mathrm{M}$ PBS. The spinal cord was removed from each rat and postfixed in 4\% PFA for 4 hours. Postfixed tissue was cryoprotected in $0.1 \mathrm{M}$ phosphate buffer ( $\mathrm{pH}$ 7.4) containing $15 \%$ and $30 \%$ sucrose solution at $4^{\circ} \mathrm{C}$. The spinal cords were embedded in OCT compound and stored at $-70^{\circ} \mathrm{C}$. To examine the cavity volume, $14 \mu \mathrm{m}$ thick serial transverse sections were prepared from $20 \mathrm{~mm}$ long spinal cord stumps ( $1 \mathrm{~mm}$ each for rostral and caudal to the lesion epicenter). Also, to compare the coexpression of various celltype-specific markers and $\mathrm{BrdU}^{+}$cells, $10 \mu \mathrm{m}$ thick serial coronal sections were prepared as described above. Coronal sections were collected from cell transplantation site to the injury epicenter sites and mounted on gelatin-coated slides.

2.7. Histology and Immunohistofluorescence. Single and double fluorescent staining was used. Single staining was used to identify newly generated cells after transplantation. For BrdU immunohistochemistry, the sections were warmed for $20 \mathrm{~min}$ and washed with $0.01 \mathrm{M}$ PBS for $10 \mathrm{~min}$. Sections were incubated in $50 \%$ formamide- $2 \mathrm{X}$ standard saline citrate at $60^{\circ} \mathrm{C}$ for $2 \mathrm{~h}$, subsequently treated with $2 \mathrm{~N} \mathrm{HCL}$ at $37^{\circ} \mathrm{C}$ for $30 \mathrm{~min}$ to denature deoxyribonucleic acid, and then incubated in $0.1 \mathrm{~mol} / \mathrm{L}$ boric acid at room temperature for $10 \mathrm{~min}$ to neutralize residual acid. The sections were incubated with rat anti-BrdU $(1: 100 ;$ Abcam, Cambridge, UK) or mouse anti-BrdU (1:100; DakoCytomation, Glostrup, Denmark). Subsequently, sections were incubated for $1 \mathrm{~h}$ at room temperature with fluorescence-conjugated secondary antibody or biotinylated antibody; the latter was reacted with avidin peroxidase for $30 \mathrm{~min}$ (ABC-kit; Vectastain Elite; Vector Laboratories, Burlingame, CA) followed by detection solution $(0.25 \mathrm{mg} / \mathrm{mL}$ diaminobenzidine, $0.03 \%$ $\mathrm{H}_{2} \mathrm{O}_{2}, 0.04 \% \mathrm{NiCl}$ ).

To determine the fate of newly generated cells after transplantation, double-fluorescent immunolabeling was performed, combining BrdU labeling with one of cell-specific phenotypic markers listed below. We used mouse anti-Nestin (1:100; Millipore, Billerica, MA) to identify neural stem progenitor; mouse anti-NG-2 chondroitin sulfate proteoglycan (anti-NG-2; 1:100; Millipore) to identify oligodendrocyte progenitor; mouse anti- $2^{\prime}, 3^{\prime}$-cyclic nucleotide $3^{\prime}$ phosphodiesterase (anti-CNPase; 1:100; Millipore), mouse antimyelin basic protein (anti-MBP, 1:100; Millipore), rabbit anti-glial fibrillary acidic protein (anti-GFAP; $1: 500$; Millipore) to identify astrocytes. After washing, samples were incubated in Alexa 488-conjugated goat anti-rat IgG ( $1: 200$; Vector Laboratories), Alexa 546-conjugated goat anti-mouse $\operatorname{IgG}(1: 200$; Vector Laboratories), or Alexa 546conjugated goat anti-rabbit IgG $(1: 200$; Vector Laboratories $)$ for $1 \mathrm{~h}$. Fluorescently stained slides were stored at $-20^{\circ} \mathrm{C}$ and observed using a fluorescence microscope equipped with a spot digital camera or a model LSM 510 confocal scanning laser microscope (Zeiss, Jena, Gemany). Apoptosis was detected by the terminal deoxynucleotidyl-transferasemediated d-UTP-biotin nick end (TUNEL) assay using the in situ cell death detection kit (Roche, Indianapolis, IN) developed using the Cy2-conjugated streptavidin (Jackson Laboratories, West Grove, PA). The slides were observed using the aforementioned confocal scanning laser microscope.

2.8. Cell Counts. The counting of $\mathrm{BrdU}^{+}$cells was done by previously described [21]. $\mathrm{BrdU}^{+}$cells were counted within a reticule of a specified area $\left(0.0682 \mathrm{~mm}^{2}\right)$ positioned in the ependymal and parenchymal region (dorsal (above the corticospinal tract), lateral, and ventromedial region of the residual white matter) in sections. White matter regions were counted in six randomly chosen sections per $1 \mathrm{~mm}^{2}$ length of spinal cord, and the numbers were averaged.

\subsection{Measurement of the Cavity Volume. For measurement of} the cavity volume, rats at 6 weeks after transplantation were used. The transverse sections were stained with hematoxylineosin (HE). The area of the cavity in the damaged spinal cord was measured in images of the sections using ImageJ version 1.38 image analyzer software (National Institutes of Health, Bethesda, MD) on consecutive sections at an interval of $70 \mu \mathrm{m}$. The volume of the cavity was then calculated by multiplying the average area by the depth of the spinal cord.

2.10. Statistical Analysis. The BBB score and cell counts were subjected to the paired $t$-test or one-way ANOVA for transplantation and PBS-treated groups of rats. Data are presented as mean \pm SE. Value of $P<0.05$ was considered statistically significant.

\section{Results}

3.1. Behavioral Assessment and Measurement of the Cavity Volume. We assessed the recovery of hindlimb function with the BBB locomotor scale from 1 day to 6 weeks after SCI. In the case of SCI rats, BBB scores were low $(<9)$. The motor function scores of MSCs-injected rats $(11.07 \pm 0.3)$ were significantly higher than the PBS-injected rats $(9.25 \pm 0.3)$ at 7 weeks after SCI. The behavioral data from the BBB locomotor scores demonstrated that MSCs-treated rats were dramatically improved in neurological function $(P<0.005$, Figure 1). In addition, the spinal cords of MSCs-injected rats had cavities much smaller than those of the PBS-injected rats. The cavity volume of MSCs-treated rat was $0.82 \pm 0.14 \mathrm{~mm}^{3}$ on average, whereas the PBS-treated rats showed a volume of $2.12 \pm 0.28 \mathrm{~mm}^{3}$. These results for cavity volume were significantly different between the MSCs-treated and PBStreated rats. Thus, MSC transplantation led to a significant improvement of behavior as well as reduction of cavity volume after SCI.

3.2. Proliferation of Endogenous Generated Cells. MSCs promoted the functional recovery and reduced the cavity volume following transplantation in SCI (Figure 1). Since an effect 


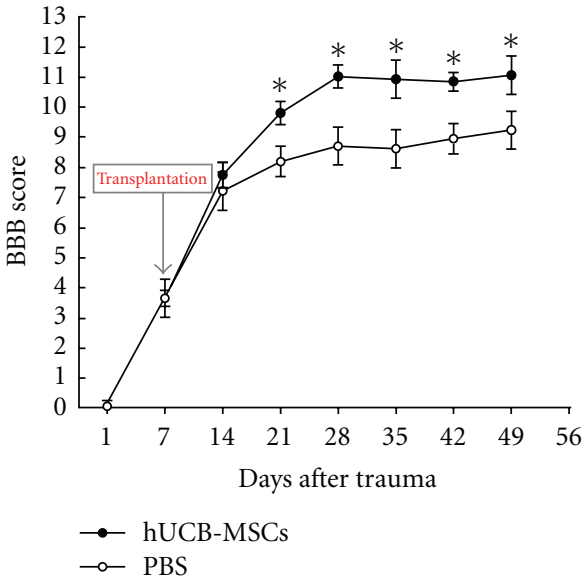

(a)

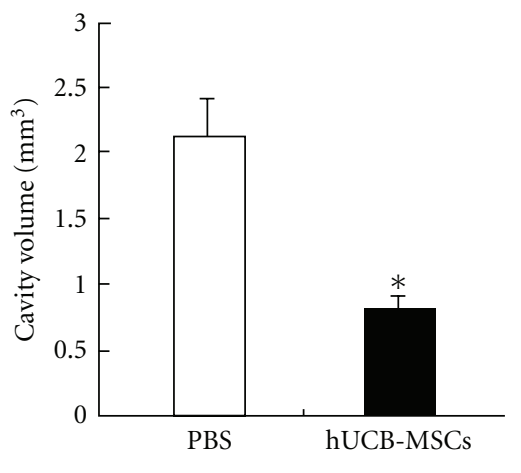

(b)

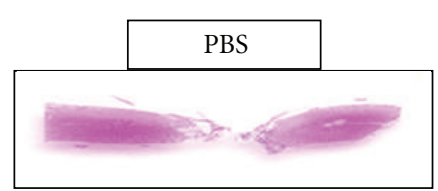

(c)

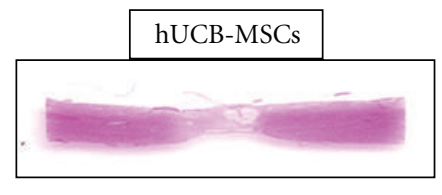

(d)

FIGURE 1: BBB scores of rats with SCI before and after hUCB-MSCs transplantation at 7 days after SCI. (a) hUCB-MSC transplantation group displayed significantly improved scores compared with control at 6 weeks after transplantation. (b) Cavity volume between the hUCB-MSC and control groups at 6 weeks after transplantation. The values of the cavity volume of the hUCB-MSC group were lower than those of the control group. (c) and (d) HE-stained sections of transplantation group and control group, $P<0.05$.

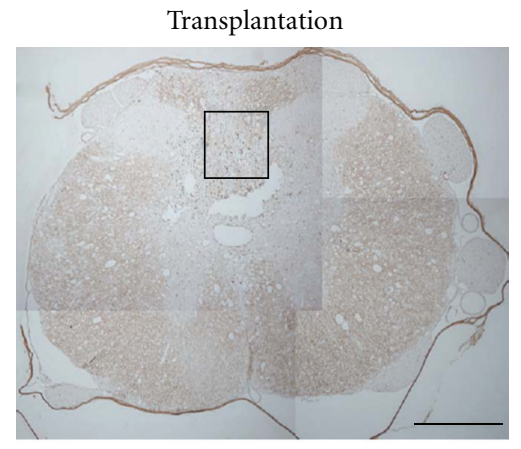

(a)

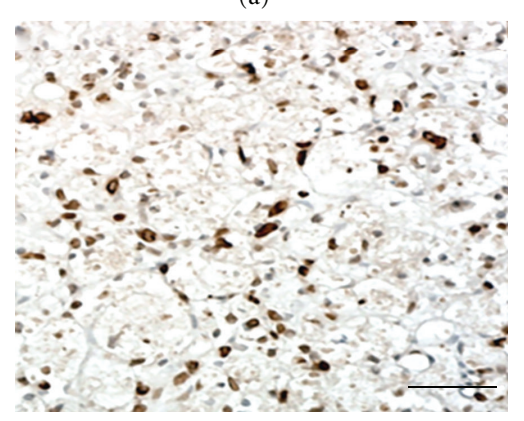

(b)

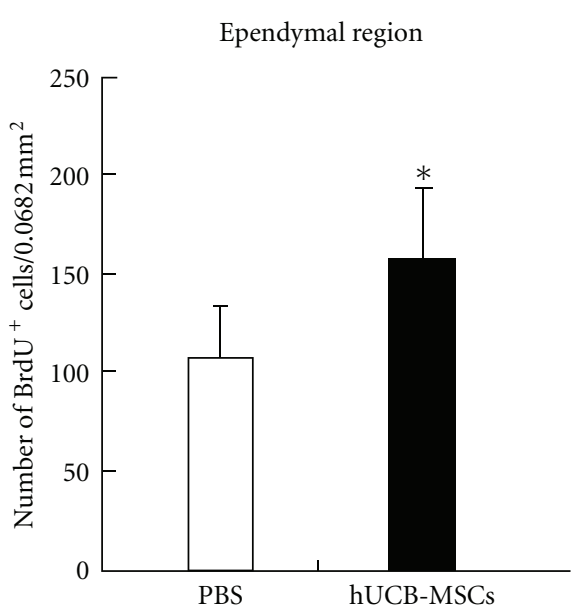

(c)

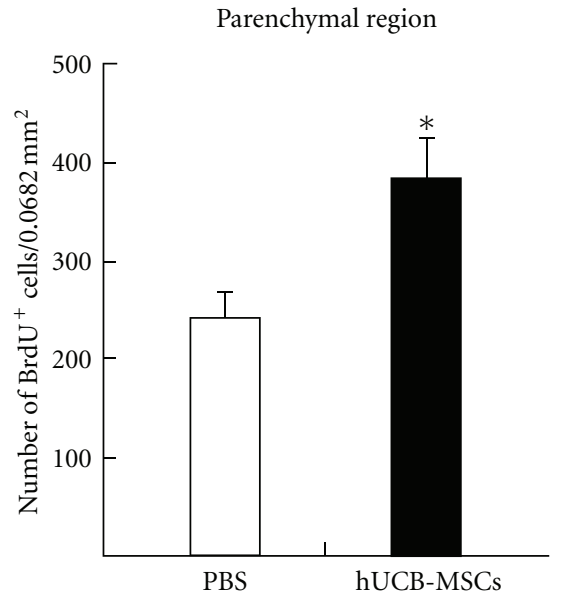

(d)

FIGURE 2: Quantitative analysis of BrdU-labeled cells in the ependymal and parenchymal regions. (a) Result of immunohistochemistry using anti-BrdU antibody. (b) Enlargement of the boxed region in (a), showing BrdU-labeled cells in the parenchymal region. (c) Average number of BrdU-labeled cells per white matter area from all five white matter areas in the parenchymal region. (d) Average number of BrdU-labeled cells per ependymal region in grey matter. At 14 days after transplantation, proliferation of endogenous cells was significantly increased from injury site to cell transplantation site in hUCB-MSCs-transplanted group compared with control group, ${ }^{*} P<0.05$.

of hUCB-MSCs was evident, we investigated whether newly generated cells were enhanced by the transplanted cells [22]. It has been suggested that oligogenesis [23] by endogenous OPCs and survival of these cells can contribute to self-repair after myelin loss [24]. With the thought that these processes might be stimulated recovery to CNS injury, an experiment was done to investigate the proliferation endogenous generated cells by daily injection of BrdU during the 7 days after transplantation. BrdU-positive cells were counted in the ependymal and parenchymal regions (Figures 2(a) and 2(b)) as previously described $[23,25]$. Proliferation of the newly generated cells increased greatly in hUCB-MSCs-treated rats 


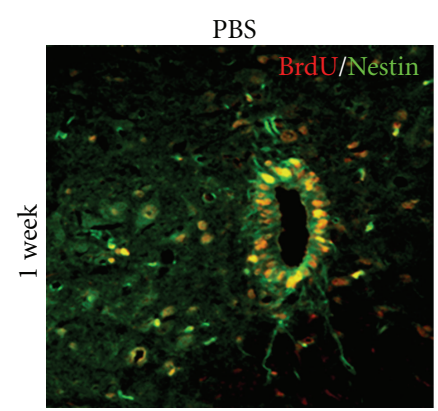

(a)

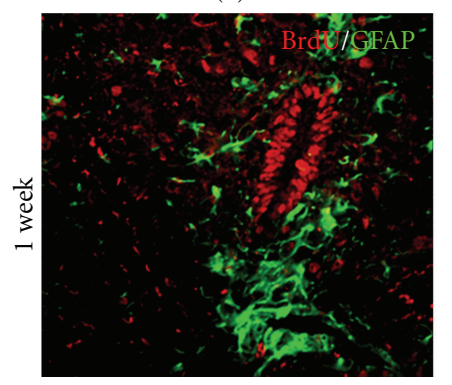

(c)

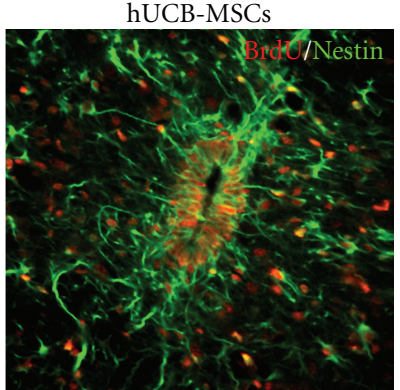

(b)

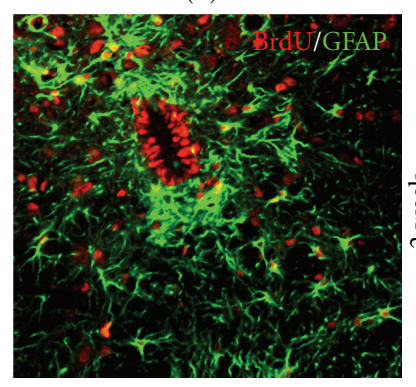

(d)

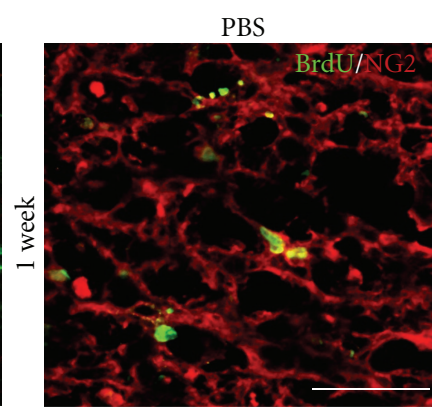

(e)

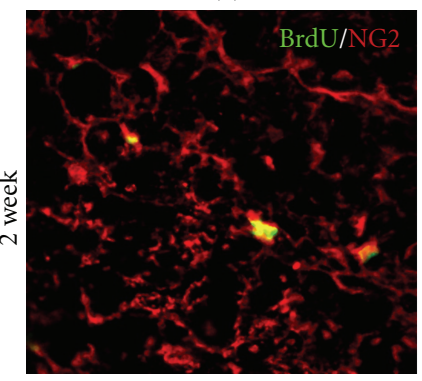

(g)

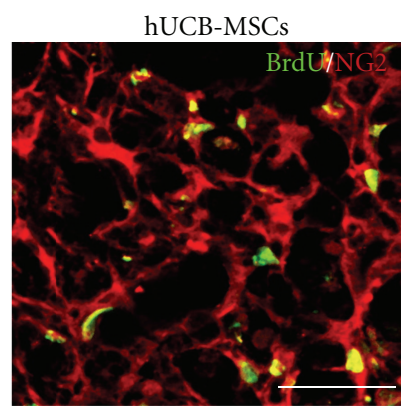

(f)

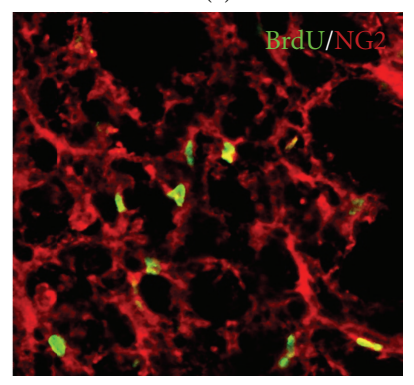

(h)

Ependymal region

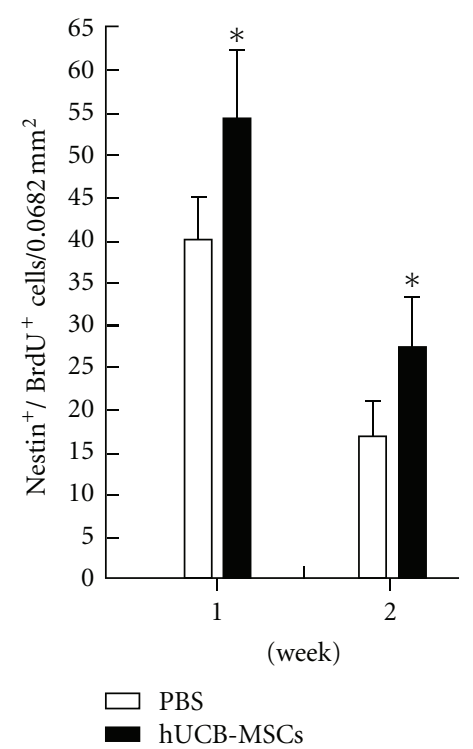

(i)

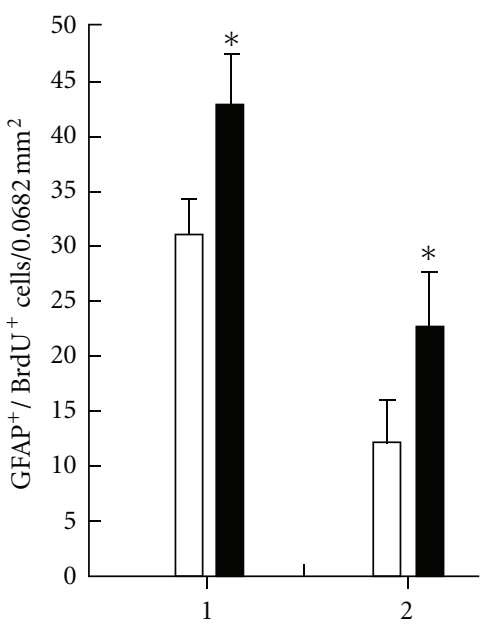

(week)

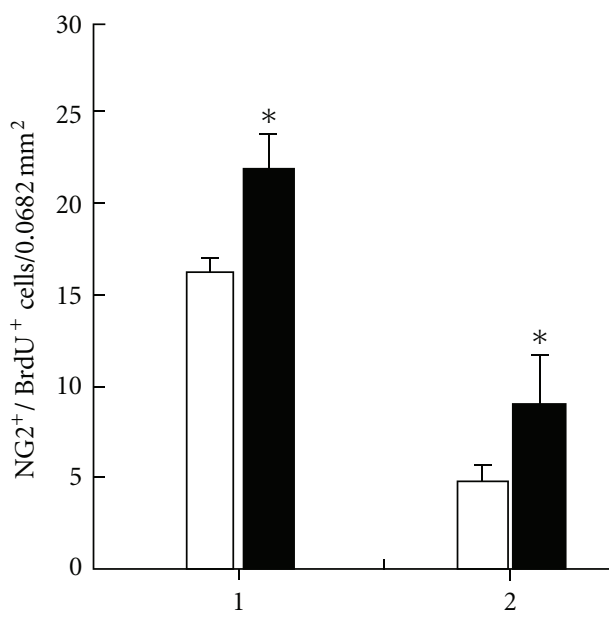

(week)

$\square$ PBS

$\square$ PBS
hUCB-MSCs

(j)

(k)

FIGURE 3: Endogenous neurogenesis induced by transplantation. Endogenous stem cells were assessed quantitatively by double staining of BrdU with nestin, GFAP, and NG2 at 1 and 2 weeks after transplantation in both the ependymal and parenchymal regions. (a)-(d) At 1 and 2 weeks following transplantation, BrdU/nestin-labeled cells as well as BrdU/GFAP-labeled astrocytes were present in ependyma. (e) and (h) BrdU-labeled NG2 cells were coexpressed at 1 and 2 weeks in the parenchyma. (i) and (j) The numbers of BrdU-labeled ependyma coexpressing GFAP/nestin were quantified at 1 and 2 weeks after transplantation. (k) The numbers of BrdU-labeled parenchyma coexpressing NG2 were quantified at 1 and 2 weeks after transplantation. ${ }^{*} P<0.05$, scale bars $=10 \mu \mathrm{m}$ in (a) $-(\mathrm{d}) ; 20 \mu \mathrm{m}$ in (e)-(h).

as compared with PBS-treated rats (Figure 2(c)). This data demonstrated that hUCB-MSCs could enhance proliferation of endogenous cells within the spinal cord.

\subsection{Characterization of Endogenous Stem Cells. Functional} recovery in response to therapeutic grafting of stem cells after SCI is related to the differentiation of grafted cells into glial cells, including astrocytes or oligodendrocytes [24]. Appropriately, an experiment was done to examine if the transplantation of MSCs could enhance the differentiation of endogenous OPCs into astrocytes or oligodendrocytes by performing immunostaining for BrdU and several phenotype markers including differentiating oligodencrocyte markers NG2, CNPase, the mature oligodendrocyte marker 
PBS

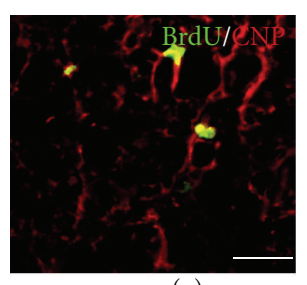

(a)

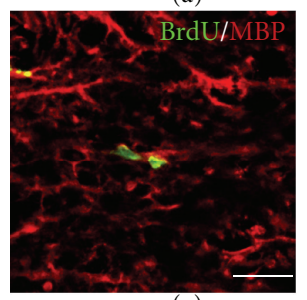

(c)

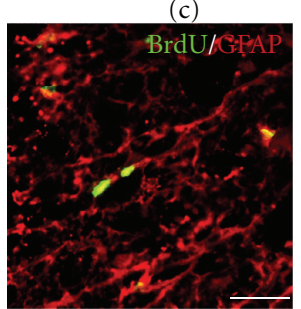

(e)

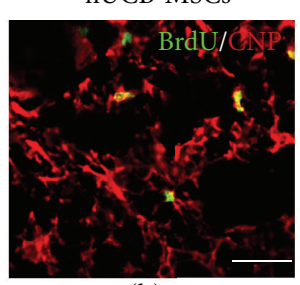

(b)
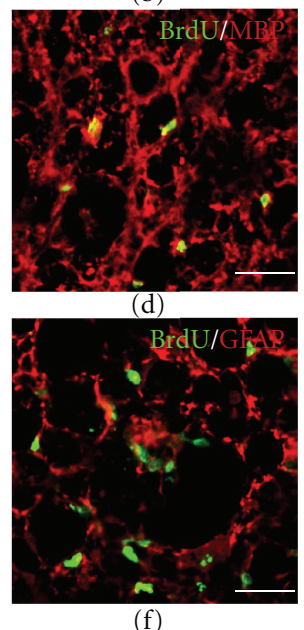

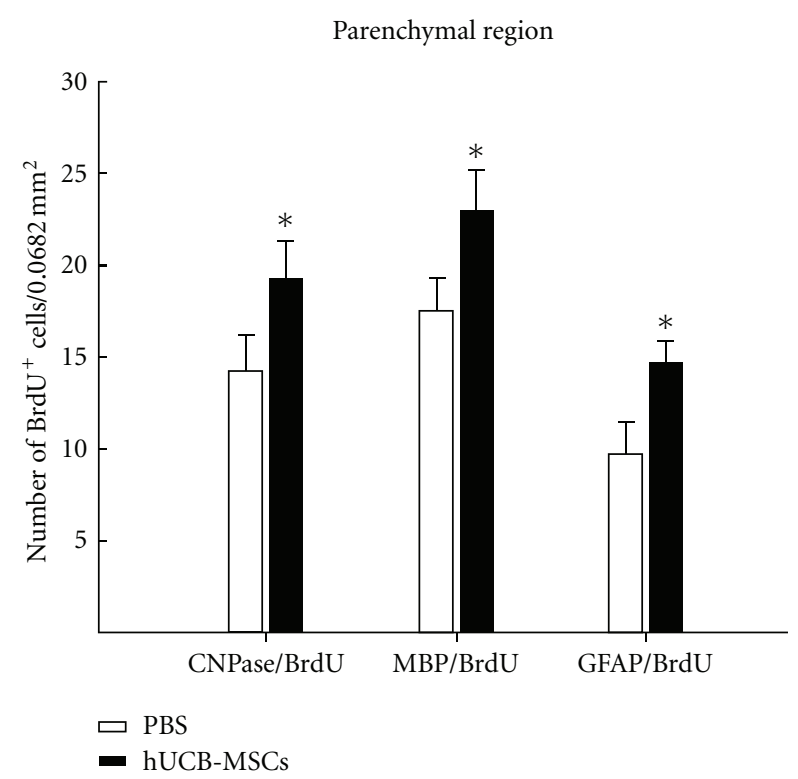

$(\mathrm{g})$

Figure 4: Quantitative analysis of endogenous oligogenesis by hUCB-MSCs. At 2 weeks after cell transplantation, BrdU and cell-specific markers were observed up to the edge of the SCI region. (a) and (b) BrdU/CNPase-labeled cells were present. (c) and (d) BrdU/MBP-labeled cells. (e) and (f) BrdU/GFAP-labeled cells. (g) Quantity of BrdU/CNPase, MBP, and GFAP-labeled cells. ${ }^{*} P<0.05$. Scale bars: $10 \mu \mathrm{m}$.

MBP, GFAP typical of astrocytes, and the neural stem cell marker nestin. Cells in the ependymal and parenchymal region were counted in sections from the injury epicenter to cell transplantation site. One and 2 weeks after cell transplantation, the numbers of BrdU positive cells were significantly increased compared with the PBS group (Figure 3). In the ependymal region, BrdU-labeled nestin and GFAP cells were increased compared with the PBS group at 1 and 2 weeks (Figure 3 ). The numbers of BrdU-labeled NG2 positive cells were also significantly increased compared with the PBS group in the parenchymal region (Figure 3). Also, BrdU-labeled cells displaying strong immunoreactivities for CNPase, MBP, or GFAP in the cell transplantation group were evident. But these immunoreactivities were weak for those rats treated with PBS (Figure 4). These data suggest that hUCB-MSCs are an influential microenvironment within the spinal cord.

3.4. Apoptotic Phenomena of Endogenous Cells. To investigate whether transplantation of MSCs have a protected injured spinal cord cells from apoptosis, a TUNEL assay was performed on sections obtained from the injury site on 2 weeks after transplantation. Numerous TUNEL-positive (green) cells were observed at the injury site in PBS-treated rats. The number of TUNEL positive cells was significantly lower in MSC-treated rats than in PBS-treated rats (Figure 5(b)). Taken together, these results indicate that hUCB-MSCs not only promote oligogenesis in the spinal cord but also have a neuroprotective effect relative with cavity volume (Figure 1(c)).

\section{Discussion}

In this study, hUCB-MSCs that were transplanted after SCI survived in and around the injured site and were able to ameliorate some of the behavior effects of SCI, as measured by spontaneous limb movement in an open-field test, hind limb extension, and toe spread. In addition, the cavities of MSC-treated rats were much smaller than PBS-injected rats. Cavity formation is a characteristic of progressive tissue necrosis, which follows the initial primary cell destruction in SCI. Therefore, reduction of the cavity volume means that transplanted MSCs after SCI have a neuroprotective effect. The presently indicated therapeutic effect of hUCB-MSCs in SCI agrees with previous data [26], but the exact mechanisms to improve the functional deficits remain to be elucidated.

A prior study showed that transplanted cells ameliorated the functional recovery through the integration into spinal cord tissue and establishment of some connections within the injured area of the spinal cord [27]. However, the transplantation of hUCB-MSCs could not solely account for functional recovery after SCI. Other possibility may be various beneficial actions of endogenous neurogenesis or oligogenesis within the adult spinal cord which is largely mediated via trophic influences. Previous studies have indicated that MSCs could produce trophic factors, cytokines, and other neuroprotective factors in stroke or traumatic brain injury $[28,29]$. These factors and cytokines can then promote the regrowth of interrupted nerve fiber tract. BMS cells secrete more than 20 cytokines in vitro, and hUCB-MSCs can secrete a number of cytokines and 


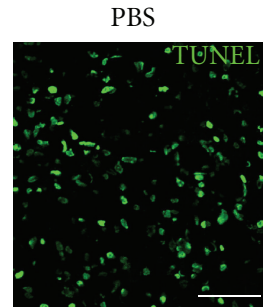

(a)

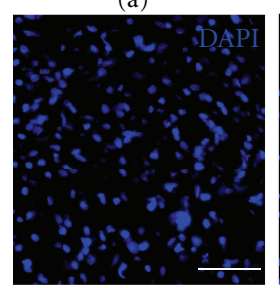

(c)

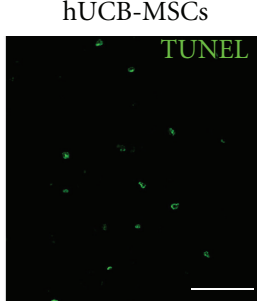

(b)

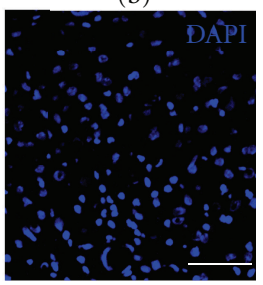

(d)

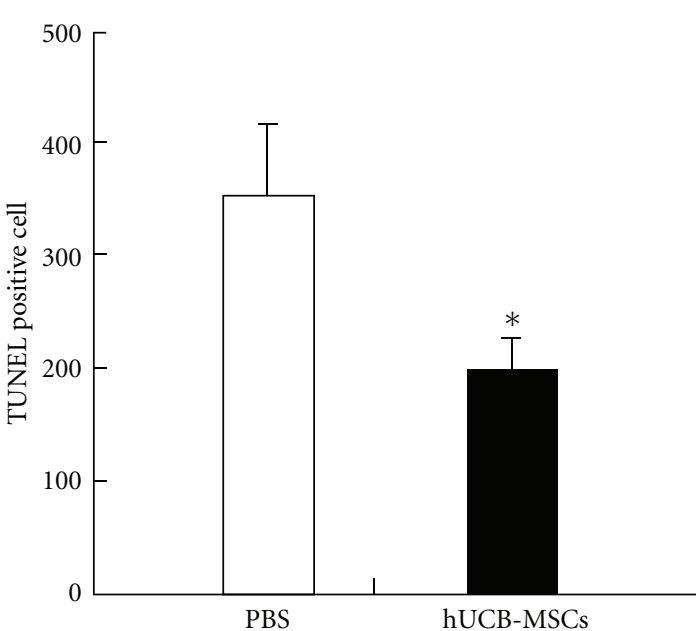

(e)

Figure 5: Protection of apoptosis by hUCB-MSCs as revealed by TUNEL assay in the injury site at 2 weeks after transplantation. (a)-(d) TUNEL staining (green) and staining with 4',6-diamidino-2-phenylindole (blue) indicate undergoing apoptotic cell death. (e) Quantity of TUNEL positive cells. The number of TUNEL positive cells was significantly reduced in cell transplantation group than in control group. ${ }^{*} P<0.05$, scale bars denote: $10 \mu \mathrm{m}$.

chemokines [30]. Therefore, these factors and some of the other cytokines secreted by hUCB-MSCs may function as survival and differentiation factors for neural progenitor cells and then play an important role in the proliferation and differentiation of neural tissue and in the increase of central nerve system plasticity $[31,32]$.

To understand whether the transplanted hUCB-MSCs are capable of restoring the production of endogenous cells, we studied the mechanisms that contributed to functional recovery by determining the endogenous cell proliferation and differentiation into glial cells following transplantation. Compared to the control group, transplanted cells increased endogenous cell division within the SCI area and a subpopulation of newly dividing cells. Also, in the received, the transplanted cells, immature and mature oligodendrocytes, and astrocytes were stimulated. These observations support the possibility that factors produced by hUCB-MSCs activate nearby oligogenesis, and that activation of the astrocytes increases in oligogenesis, since astrocytes are located in close proximity to neural stem cells and express several factors that independently increase oligogenesis. In addition, some of transplanted cells were BrdU-positive cell. It has been shown that transplanted cells might proliferate in the spinal cord. But, these cells are not differentiated neural lineage markers. In agreement with the present findings, a previous study reported not only extensive oligogenesis of newly born cells after SCI but also that MSCs promote oligogenesis in neural stem cells in vitro $[24,33]$.

Presently, the majority of hUCB-MSCs progressed to apoptotic cell death. However, MSC-treated rats displayed markedly reduced apoptotic cell death in the injured site. These results suggest that functional recovery might result in endogenous oligogenesis and neuroprotection stimulated by trophic factors secreted into transplanted cells.
The collective results support the view that hUCB-MSCs transplantation is beneficial in SCI by virtue of their growth factor secretion and ability to provide physical support to growing axons. Further studies are needed to confirm that the benefit obtained from hUCB-MSCs persists at later time points and/or to improve the efficacy of the transplanted hUCB-MCSs. Also, the mechanisms underlying functional recovery after transplantation of hUCB-MSCs remain to be further investigated.

\section{Conclusion}

We have shown that stem cell therapy of hUCB-MSCs may provide more of functional recovery in spinal cord injury such as reduction of cavity volume, increasing of cell proliferation and endogenous oligogenesis, and decreasing of apoptosis. Therefore, the author suggests that promotion of oligogenesis by hUCB-MSCs may provide a scientific basis for the potential use of these cells as a therapeutic tool for the treatment of other disease.

\section{Conflict of Interests}

The authors declare that there is no conflict of interests.

\section{Acknowledgments}

This study was supported by the Basic Science Research program through the National Research Foundation of Korea (NRF) funded by the Ministry of Education, Science and Technology (2010-0022845), Republic of Korea. This study was supported by a grant of the Korea Healthcare technology R\&D Project, Ministry of Health, Welfare\& Family Affairs, Republic of Korea (A092258). 


\section{References}

[1] M. P. Kurnellas, A. Nicot, G. E. Shull, and S. Elkabes, "Plasma membrane calcium ATPase deficiency causes neuronal pathology in the spinal cord: a potential mechanism for neurodegeneration in multiple sclerosis and spinal cord injury," FASEB Journal, vol. 19, no. 2, pp. 298-300, 2005.

[2] J. W. McDonald and V. Belegu, "Demyelination and remyelination after spinal cord injury," Journal of Neurotrauma, vol. 23, no. 3-4, pp. 345-359, 2006.

[3] L. J. Rosenberg and J. R. Wrathall, "Quantitative analysis of acute axonal pathology in experimental spinal cord contusion," Journal of Neurotrauma, vol. 14, no. 11, pp. 823-838, 1997.

[4] L. Brundin, H. Brismar, A. I. Danilov, T. Olsson, and C. B. Johansson, "Neural stem cells: a potential source for remyelination in neuroinflammatory disease," Brain Pathology, vol. 13, no. 3, pp. 322-328, 2003.

[5] G. Martino and S. Pluchino, "The therapeutic potential of neural stem cells," Nature Reviews Neuroscience, vol. 7, no. 5, pp. 395-406, 2006.

[6] O. Lindvall and Z. Kokaia, "Stem cells for the treatment of neurological disorders," Nature, vol. 441, no. 7097, pp. 10941096, 2006.

[7] S. Kabatas and Y. D. Teng, "Potential roles of the neural stem cell in the restoration of the injured spinal cord: review of the literature," Turkish Neurosurgery, vol. 20, no. 2, pp. 103-110, 2010.

[8] T. L. Ben-Shaanan, T. Ben-Hur, and J. Yanai, “Transplantation of neural progenitors enhances production of endogenous cells in the impaired brain," Molecular Psychiatry, vol. 13, no. 2, pp. 222-231, 2008.

[9] M. F. Pittenger, A. M. Mackay, S. C. Beck et al., "Multilineage potential of adult human mesenchymal stem cells," Science, vol. 284, no. 5411, pp. 143-147, 1999.

[10] Y. Ogawa, K. Sawamoto, T. Miyata et al., "Transplantation of in vitro-expanded fetal neural progenitor cells results in neurogenesis and functional recovery after spinal cord contusion injury in adult rats," Journal of Neuroscience Research, vol. 69, no. 6, pp. 925-933, 2002.

[11] Y. I. Tarasenko, J. Gao, L. Nie et al., "Human fetal neural stem cells grafted into contusion-injured rat spinal cords improve behavior," Journal of Neuroscience Research, vol. 85, no. 1, pp. 47-57, 2007.

[12] S. E. Yang, C. W. Ha, M. H. Jung et al., "Mesenchymal stem/progenitor cells developed in cultures from UC blood," Cytotherapy, vol. 6, no. 5, pp. 476-486, 2004.

[13] P. R. Sanberg, A. E. Willing, S. Garbuzova-Davis et al., "Umbilical cord blood-derived stem cells and brain repair," Annals of the New York Academy of Sciences, vol. 1049, pp. 6783, 2005.

[14] J. R. Pineda, N. Rubio, P. Akerud et al., "Neuroprotection by GDNF-secreting stem cells in a Huntington's disease model: optical neuroimage tracking of brain-grafted cells," Gene Therapy, vol. 14, no. 2, pp. 118-128, 2007.

[15] J. Chen, Y. Li, M. Katakowski et al., "Intravenous bone marrow stromal cell therapy reduces apoptosis and promotes endogenous cell proliferation after stroke in female rat," Journal of Neuroscience Research, vol. 73, no. 6, pp. 778-786, 2003.

[16] K. C. Wollert and H. Drexler, "Clinical applications of stem cells for the heart," Circulation Research, vol. 96, no. 2, pp. 151$163,2005$.
[17] P. J. Horner and F. H. Gage, "Regenerating the damaged central nervous system," Nature, vol. 407, no. 6807, pp. 963970, 2000.

[18] B. H. Dobkin and L. A. Havton, "Basic advances and new avenues in therapy of spinal cord injury," Annual Review of Medicine, vol. 55, pp. 255-282, 2004.

[19] D. M. Basso, M. S. Beattie, and J. C. Bresnahan, "Graded histological and locomotor outcomes after spinal cord contusion using the NYU weight-drop device versus transection," Experimental Neurology, vol. 139, no. 2, pp. 244-256, 1996.

[20] D. M. Basso, M. S. Beattie, and J. C. Bresnahan, "A sensitive and reliable locomotor rating scale for open field testing in rats," Journal of Neurotrauma, vol. 12, no. 1, pp. 1-21, 1995.

[21] L. J. Zai and J. R. Wrathall, "Cell proliferation and replacement following contusive spinal cord injury," Glia, vol. 50, no. 3, pp. 247-257, 2005.

[22] S. W. Yoo, S. S. Kim, S. Y. Lee et al., "Mesenchymal stem cells promote proliferation of endogenous neural stem cells and survival of newborn cells in a rat stroke model," Experimental and Molecular Medicine, vol. 40, no. 4, pp. 387-397, 2008.

[23] L. J. Rosenberg, L. J. Zai, and J. R. Wrathall, "Chronic alterations in the cellular composition of spinal cord white matter following contusion injury," Glia, vol. 49, no. 1, pp. 107-120, 2005.

[24] H. Yang, P. Lu, H. M. McKay et al., "Endogenous neurogenesis replaces oligodendrocytes and astrocytes after primate spinal cord injury," Journal of Neuroscience, vol. 26, no. 8, pp. $2157-$ 2166, 2006.

[25] L. J. Zai, S. Yoo, and J. R. Wrathall, "Increased growth factor expression and cell proliferation after contusive spinal cord injury," Brain Research, vol. 1052, no. 2, pp. 147-155, 2005.

[26] C. P. Hofstetter, E. J. Schwarz, D. Hess et al., "Marrow stromal cells form guiding strands in the injured spinal cord and promote recovery," Proceedings of the National Academy of Sciences of the United States of America, vol. 99, no. 4, pp. 21992204, 2002.

[27] Z. M. Zhao, H. J. Li, H. Y. Liu et al., "Intraspinal transplantation of CD34+ human umbilical cord blood cells after spinal cord hemisection injury improves functional recovery in adult rats," Cell Transplantation, vol. 13, no. 2, pp. 113-122, 2004.

[28] J. R. Munoz, B. R. Stoutenger, A. P. Robinson, J. L. Spees, and D. J. Prockop, "Human stem/progenitor cells from bone marrow promote neurogenesis of endogenous neural stem cells in the hippocampus of mice," Proceedings of the National Academy of Sciences of the United States of America, vol. 102, no. 50, pp. 18171-18176, 2005.

[29] R. McKay, "Stem cells in the central nervous system," Science, vol. 276, no. 5309, pp. 66-71, 1997.

[30] M. B. Newman, A. E. Willing, J. J. Manresa, C. D. Sanberg, and P. R. Sanberg, "Cytokines produced by cultured human umbilical cord blood (HUCB) cells: implications for brain repair," Experimental Neurology, vol. 199, no. 1, pp. 201-208, 2006.

[31] A. Erices, P. Conget, and J. J. Minguell, "Mesenchymal progenitor cells in human umbilical cord blood," British Journal of Haematology, vol. 109, no. 1, pp. 235-242, 2000.

[32] Y. Li, J. Chen, X. G. Chen et al., "Human marrow stromal cell therapy for stroke in rat: neurotrophins and functional recovery," Neurology, vol. 59, no. 4, pp. 514-523, 2002.

[33] Q. M. Li, Y. M. Fu, Z. Y. Shan et al., "MSCs guide neurite directional extension and promote oligodendrogenesis in NSCs," Biochemical and Biophysical Research Communications, vol. 384, no. 3, pp. 372-377, 2009. 

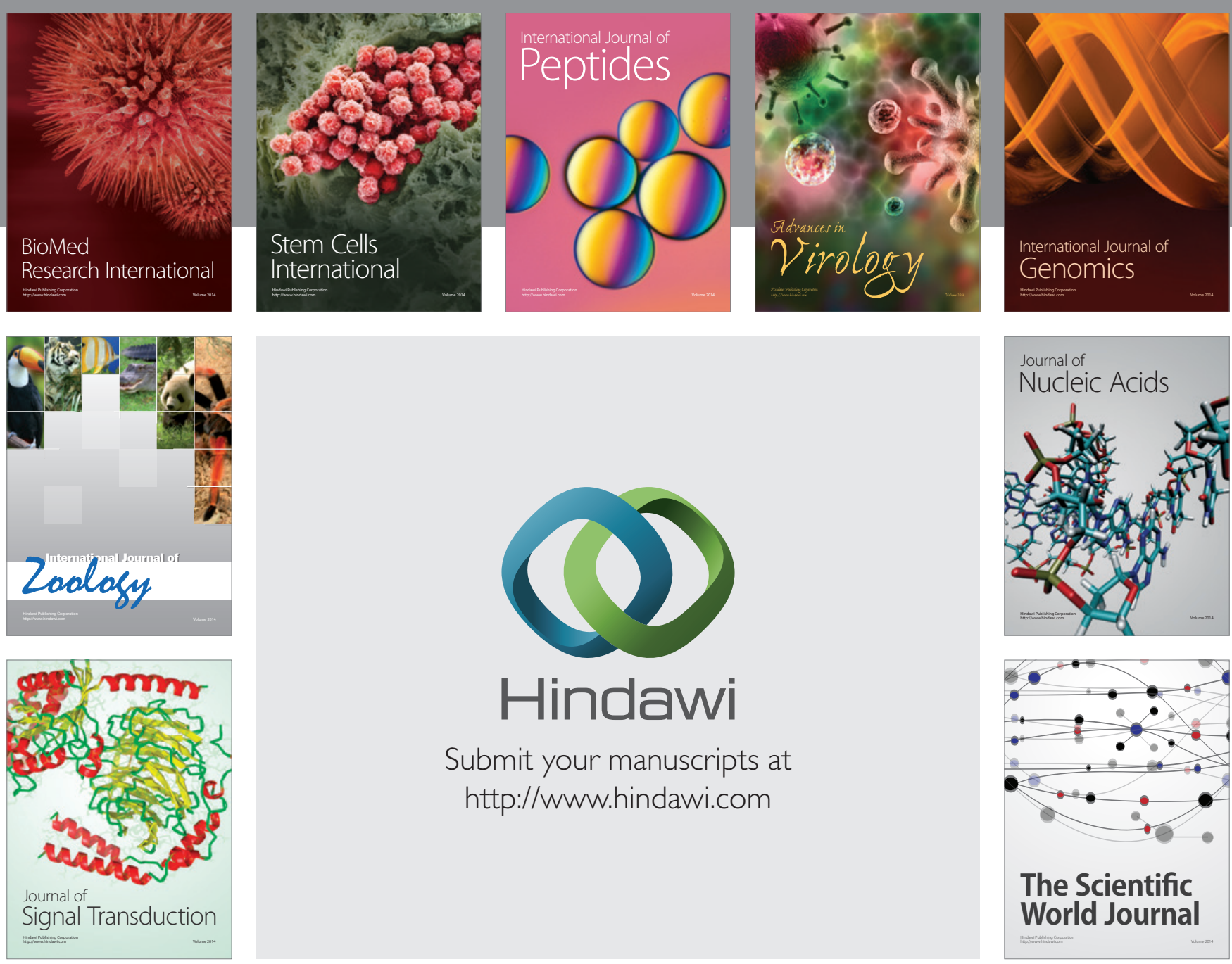

Submit your manuscripts at

http://www.hindawi.com
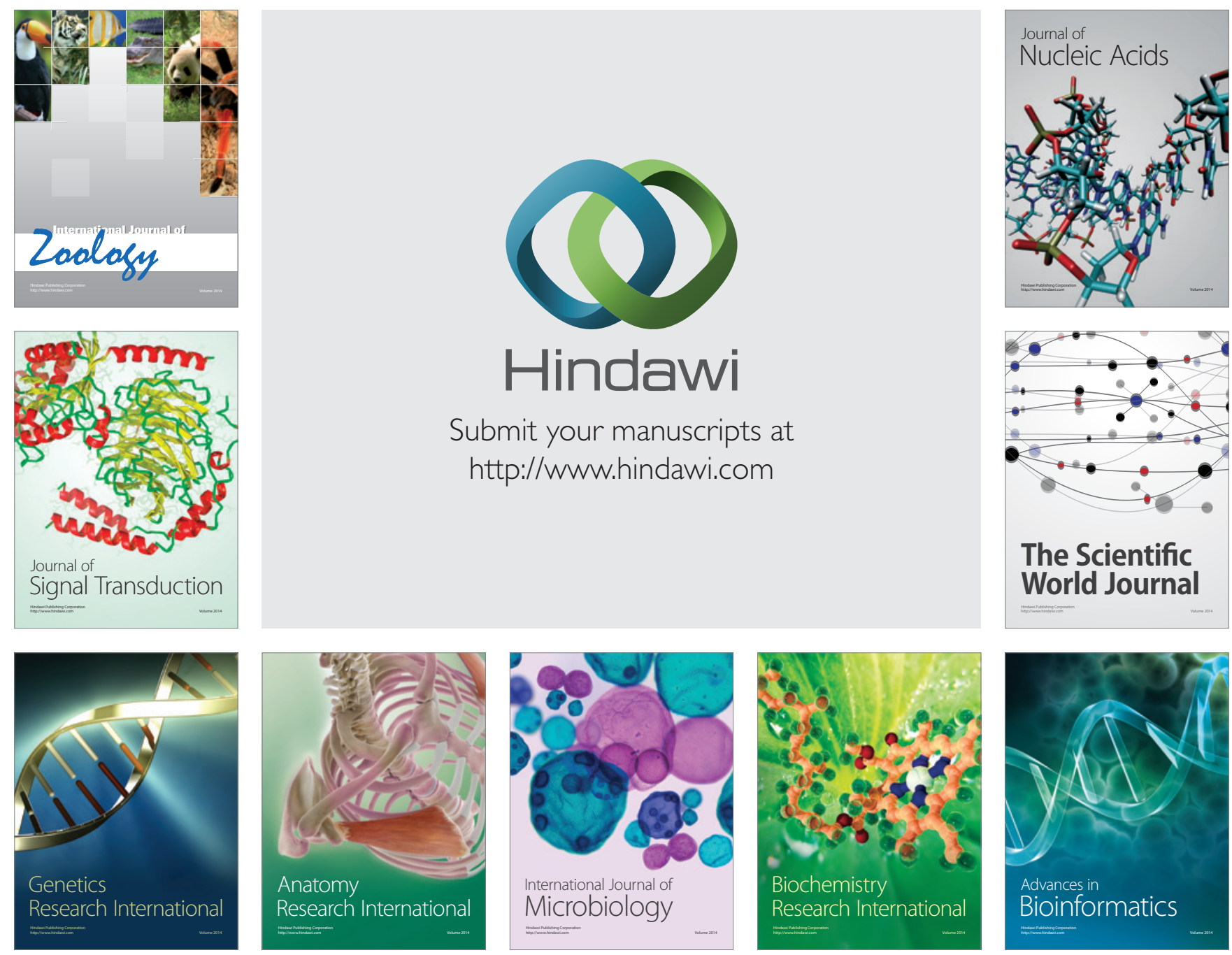

The Scientific World Journal
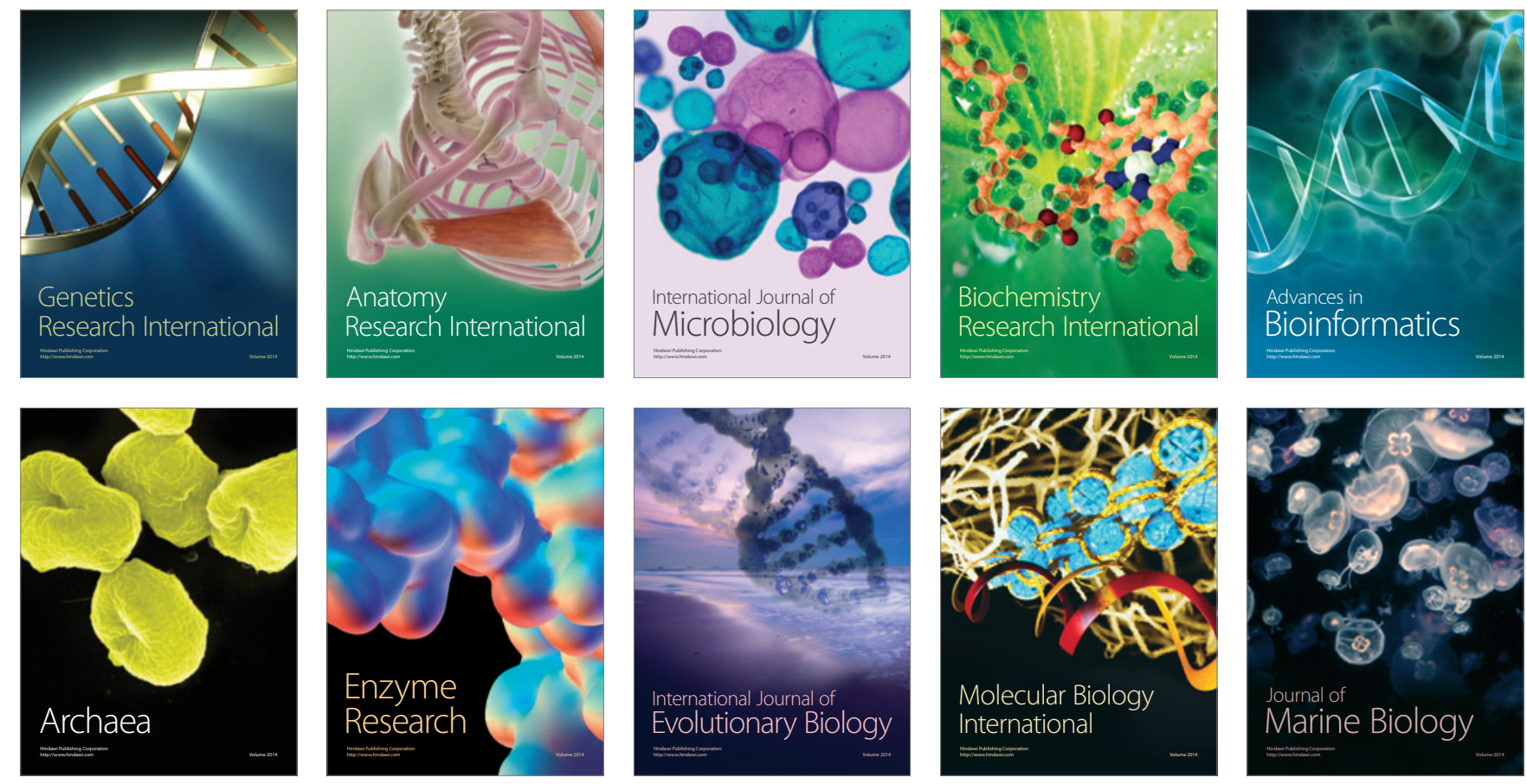